\title{
Letter to the Editor :
}

Title: Resorbable magnesium scaffolds; the learning curve continues...

Regarding "Magmaris very late in-scaffold restenosis: Has the "black boxes" nightmare come back?" Cath Cardiovasc Interv 2019; Nov 2019 (online)

Johan Bennett, MD PhD, Keir McCutcheon, MD, PhD.

Department of Cardiovascular Medicine, University Hospitals Leuven, Leuven, Belgium

Text word count: (including text, references, and figure legends): 1039

Figures: 0

References: 9

Indexing words: resorbable magnesium scaffolds, optical coherence tomography, scaffold collapse

"The authors declare no conflict of interest"

Johan Bennett has received research grants from Abbott Vascular and Biotronik AG, and speaking \& consultancy fees from Abbott Vascular, Biotronik AG and Boston Scientific

Address for correspondence:

Prof. Dr. J. Bennett, Department of Cardiovascular Medicine, University Hospitals Leuven

Herestraat 49, B 3000 Leuven, Belgium

Tel: +32-16-344235; fax:+32-16-344240

e-mail:johan.bennett@uzleuven.be 
We read with interest the publication by Bayon et al.(1) where a case of very-late resorbable magnesium scaffold (RMS) restenosis was reported. In this case very late restenosis occurred 20 months post implantation with the authors attributing this to in-scaffold "neo tissue" growth.

Bioresorbable scaffold technology provides transient vessel support with drug-delivery capability without the long-term limitations of permanent metallic drug-eluting stents (DES). The sirolimus-eluting resorbable magnesium scaffold Magmaris (Biotronik AG, Bulach, Switzerland), the only metallic CE-marked resorbable scaffold available, provides vessel support for up to 3 months and resorbs within 1 year (2). The first clinical studies testing this device in mainly simple coronary artery lesions have shown promising results $(3,4)$. However, there have been some reports of potential early scaffold collapse due to insufficient lasting radial strength $(5,6)$. Therefore, it is extremely important that patient selection and implantation technique follow expert recommendations (7).

In this case the authors are to be congratulated for the meticulous vessel preparation and the use of intracoronary imaging to assess the final result. Nevertheless, in order to educate the wider interventional community, we would like to draw the attention to several technical as pects of this case that are best considered in order to improve the safety and efficacy of this novel technology.

1. In our experience, the implantation of $50 \mathrm{~mm}$ of scaffold precisely at the location of a hinge point in the right coronary artery (RCA) predisposes to early scaffold collapse. The magnesium scaffold provides scaffolding up to 3 months predisposing to radial collapse and even in metal stents, the hinge point is known to increase the incidence of stent fracture and in-stent restenosis $(8,9)$.

2. According to expert recommendations (7), short lesions covered with a single scaffold are ideal lesions for RMS. However, if a second scaffold is required (for example, because of edge dissection), this should ideally be placed in a "side by side" manner. Upfront implantation of overlapping long scaffolds is best avoided.

3. Although post implantation OCT revealed excellent strut apposition, there was also clear underexpansion present (from 6 o'clock to 12 o'clock in Figure 1b), and this is exactly at the point of the overlapping scaffolds (Lumen area $9.9 \mathrm{~mm}^{2}$, Lumen mean diameter $3.54 \mathrm{~mm}$ in proximal scaffolded segment vs Lumen area $8.8 \mathrm{~mm}^{2}$, Lumen 
mean diameter $3.34 \mathrm{~mm}$ in segment of overlapping scaffold, respectively). More aggressive pre- and post-dilatation might therefore have been advised.

It is our opinion that the presence of overlapping and underexpanded scaffolds, at the site of a coronary hinge point in the RCA, predisposed to a degree of scaffold collapse, which surprisingly only became symptomatic a number of months later. The OCT at 20 months indeed shows clear luminal narrowing with remnants of the magnesium scaffold evidenced by the footprint of the amorphous calcium phosphate, with some immature neointima. However, to term this the "return of the black box nightmare" is maybe a little rushed. On OCT the amorphous calcium phosphate appears as opaque round structures, which could give the impression of "black boxes" sandwiched between these structures. Unlike the black boxes typical of bioresorbable scaffold devices such as Absorb (Abbott, Santa Clara, CA), voids in the arterial wall are not typical of the healing process after RMS implantation.

An equivalent of scaffold collapse can probably be considered stent fracture in metallic DES. In a meta-analysis of 8 studies ( $n=5,321$ patients) within $<12$ months follow-up after DES implantation, the mean reported incidence of stent fracture was $4.0 \%$, with the probability of stent fracture being highest in the RCA, lesions with overlapping stents, and longer stents (average length $46 \mathrm{~mm}$ ) (8). Similarly, in a large registry of stent fracture in 4 centers in China, multivariate analysis revealed that independent risk factors for stent fracture included stents in the RCA, stainless steel stents, stent length $>25 \mathrm{~mm}$, hinge motion (in any of the epicardial arteries), overlapping stents and multiple stents (9).

We are of the opinion, and recent data would suggest, that there remains a definite role for RMS in the treatment of simple coronary artery disease, especially in young patients. It is important however, that the wider interventional community is aware of the limitations of the current generation bioresorbable technology. Operators should be cognizant of the recommendations for the use of bioresorbable scaffolds, be it magnesium or poly-L lactic acid based scaffolds, and to especially pay attention to the avoidance of underexpansion, overlapping scaffolds and the implantation at coronary hinge sites. 


\section{References}

1. Bayón J, Santás-Álvarez M, Ocaranza-Sánchez R, González-Juanatey C. Magmaris very late in-scaffold restenosis: Has the "black boxes" nightmare come back?. Cath Cardiovasc Interv November 25th 2019

2. Bennett J, De Hemptinne Q, McCutcheon K, Magmaris resorbable magnesium scaffold for the treatment of coronary heart disease: overview of its safety and efficacy. Expert Rev Med Devices 2019; 16(9):757-769.

3. Testa L, De Carlo M, Petrolini A, Rapetto C, Varbella F, Cortese B, et al. Sustained safety and clinical performance of a drug-eluting absorbable metal scaffold up to 24 months: Pooled outcomes of BIOSOLVE-II and BIOSOLVE-III. Eurolntervention 2017;13:432439.

4. Verheye S, Wlodarczak A, Montorsi P, et al. Safety and performance of a resorbable magnesium scaffold under real-world conditions: 12-month outcomes of the first 400 patients enrolled in the BIOSOLVE-IV registry. Eurolntervention 2019; doi: 10.4244/EIJD-18-01058. [Epub ahead of print]

5. Marynissen T, McCutcheon K, Bennett J. Early collapse causing stenosis in a resorbable magnesium scaffold. Catheter Cardiovasc Interv. 2018; 92( 2): 310- 312.

6. Barkholt TO, Neghabat O, Terkelsen CJ, Christiansen EH, Holm NR. Restenosis in a collapsed magnesium bioresorbable scaffold. Circ Cardiovasc Interv 2017;10:e005677.

7. Fajadet $J$, Haude $M$, Joner $M$, et al. Magmaris preliminary recommendation upon commercial launch: a consensus from the expert panel on 14 April 2016. Eurolntervention 2016; 12(7): 828-33.

8. Chakravarty $\mathrm{T}^{1}$, White AJ, Buch M, Naik H, Doctor N, Schapira J, Kar S, Forrester JS, Weiss RE, Meta-analysis of incidence, clinical characteristics and implications of stent fracture. Am J Cardiol. 2010 Oct 15;106(8):1075-80.

9. Kan J, Ge Z, Zhang JJ, Liu ZZ, Tian NL, Ye F, Li SJ, Qian XS, Yang S, Chen MX, Rab T, Chen SL, et al. Incidence and Clinical Outcomes of Stent Fractures on the Basis of 6,555 Patients and 16,482 Drug-Eluting Stents From 4 Centers. JACC Cardiovasc Interv. 2016 Jun 13;9(11):1115-23 